\title{
Trigeminal Ganglion Axons Are Repelled By Their Presumptive Targets
}

\author{
M. William Rochlin and Albert I. Farbman \\ Department of Neurobiology and Physiology, Northwestern University, Evanston, Illinois 60208-3520
}

Previous work suggested that in mouse, presumptive targets of the trigeminal ganglion, rather than intermediate structures, attract pioneer axons from the time their growth cones exit the ganglion (Lumsden and Davies, 1986). In rat we find that some presumptive targets repel trigeminal axons. The repellant activity is concentrated in the anterior and ventral epithelium of the mandibular arch at embryonic day 12 (E12) and was also present in the maxillary arch. The activity is blocked by antineuropilin-1. E13 mandible explants repel trigeminal axons during the first day of outgrowth in vitro, but thereafter permit or attract trigeminal ganglion axon outgrowth. By E14, lingual nerve afferents first enter the tongue in vivo, and the repellant influence becomes restricted to the midline. The progressive restriction of the repellant influence may contribute to the in vivo progression of nerve development: the earliest afferents turn anteriorly lateral to the tongue, but subsequently arriving afferents advance into the tongue and then turn away from the midline. Thus, the repellant may influence the order of nerve branch development and the timing of innervation of epithelial and subepithelial targets. Heterochronic studies revealed that the loss of repellant influence from presumptive lateral tongue surface results from downregulation of the repellant activity, not of responsiveness to the repellant. Because presumptive targets repel trigeminal axons during the initial stages of advance from the trigeminal ganglion and do not have a net attractive influence until after afferents have arrived near the target, intermediate structures must guide these axons initially.

Key words: chemorepellant; chemoattractant; axon guidance; peripheral nervous system; trigeminal ganglion; mandible; development; neuropilin-1; semaphorin III/D
We are investigating guidance mechanisms that influence the trajectory of lingual sensory axons as they navigate toward the tongue during embryonic development. The tongue is unusual in that sensory axons arrive before motor axons, and therefore have a pioneering role (Hogg and Bryant, 1969; Moody and Heaton, 1983). As an initial step, we have investigated whether trigeminal axons are influenced by diffusible guidance factors secreted from presumptive target tissues. In addition, we describe the early development of mandibular and lingual innervation.

A variety of axon guidance systems (Tessier-Lavigne and Goodman, 1996) are evident in the developing mandible (arch Ib). Short-range cues, including extracellular matrix molecules laminin and tenascin (Riggott and Moody 1987; Mistretta and Haus, 1996), and ephrin receptors and ligands (Gale et al., 1996; Flenniken et al., 1996) are present during mandibular nerve development. Long-range diffusible attractants (Lumsden and Davies, 1983, 1986; cf. Livesey and Hunt; 1997) and chemorepellants (Wright et al., 1995; Adams et al., 1996; Giger et al., 1996; Taniguchi et al., 1997; Kobayashi et al., 1997) are secreted by developing mandible during innervation. Because Lumsden and Davies $(1983,1986)$ carefully demonstrated that the chemoattrac-

\footnotetext{
Received April 7, 1998; revised June 8, 1998; accepted June 9, 1998.

This work was supported by Research and Training Center Grant 5P60DC02764 from the National Institute on Deafness and Other Communication Disorders to A.I.F. This work is dedicated in gratitude to Dr. Burt Evans, whose battle with amyotrophic lateral sclerosis never interfered with his desire to discover or his eagerness to help others. We also note that Drs. Robert O'Connor and Marc Tessier-Lavigne independently discovered what appears to be an identical phenomenon in the mouse trigeminal system, and we thank them and their colleague Zhigang He for providing anti-neuropilin-1. Finally, we thank Timothy Cho, whose expertise in computer matters was extremely helpful.

Correspondence should be addressed to M. William Rochlin, Department of Neurobiology and Physiology, Northwestern University, 2153 North Campus Drive, Hogan 2-160, Evanston, IL 60208-3520.

Copyright (C) 1998 Society for Neuroscience $\quad 0270-6474 / 98 / 186840-13 \$ 05.00 / 0$
}

tant was capable of acting over the distances that separated the target from the ganglion, the prevailing view has been that targetderived chemotropic factors, not intermediate cues, attract axons from the ganglion to the target. Recently, knock-outs of the chemorepellant semaphorin III/D or its receptor, neuropilin-1, dramatically illustrated a role for this chemorepellant system in limiting the exploration of trigeminal axons within the developing branchial arches (Kituskawa et al., 1997; Taniguchi et al., 1997). To clarify the roles of these long-range systems, we have directly tested whether presumptive target tissue has a net attractant or repellant influence on trigeminal ganglion growth cones at early to intermediate stages of mandibular nerve development [embryonic days 12-14 (E12-E14)] in rat.

Our results cast doubt on a prominent role for target-derived chemoattraction before the arrival of lingual nerve afferents within the tongue mesenchyme. Furthermore, because knockouts of genes encoding guidance molecules may only expose the earliest necessary role of those guidance molecules, we compare the development of mandible innervation with the spatiotemporal regulation of the repellant influence. This enables us to infer specific functions for the repellant influence that were not detected in the knock-out studies. Our observations suggest roles for chemorepellants in controlling peripheral nerve branch development.

\section{MATERIALS AND METHODS}

Trigeminal ganglia were dissected from rat embryos ranging in age from E12 to E14 (see Fig. 1). We define E1 as the day the dam is spermpositive. We observed variability in the sizes of embryos within individual litters. In initial experiments, care was taken to discard embryos that were overtly different in size because we could not be sure if these differences were attributable to developmental differences or other factors. In later experiments, we counted somites and pooled embryos that were \pm 1 somite of the most common stage. However, we found no 
obvious differences in the response of trigeminal ganglion explants to target explants that were resolved by this more refined developmental staging, so data are presented according to the embryonic date. At E12, the posterior half of the ganglion was cultured, and at later stages the posterolateral aspect of the ganglion (that which gives rise to the mandibular nerve) was cultured.

In most experiments, ganglion explants were cocultured with arch Ib or dorsoanterior tongue explants. From E12 and E13 embryos, a parasagittal section of arch Ib 150-200 $\mu \mathrm{m}$ in thickness was used (see Fig. $1 \mathrm{~B}$, vertical lines). The section was obtained just lateral to ( $100 \mu \mathrm{m}$ from) the medial tip of arch Ib, so it did not include the tip. From E14 embryos, medial dorsal tongue explants consisted of a strip of $\sim 200 \mu \mathrm{m}$ width containing the septum. Lateral tongue explants consisted of the adjacent dorsal surface of the tongue. For both medial and lateral tongue explants, most of the mesenchyme was removed, but a strip of mesenchyme remained, leading to a thickness of $\sim 150 \mu \mathrm{m}$. To determine the distribution of the repellant within E12 arch Ib explants, thicker sections (300 $\mu \mathrm{m}$ wide) were used. Epithelium was removed principally by dissection. After dissecting the explant, the epithelium was severed on the ventral side of the explant. One of the cut ends was pinned down to the dish, and a scalpel blade was used to roll the disk of mesenchyme off of the epithelium. Although this resulted in physical stress to the epithelium, we found that trypsin treatment, and to a lesser extent EDTA, appeared to decrease the health of the explants. Dissections were performed in Dulbecco's PBS (Life Technologies, Gaithersburg, MD), and tissue fragments were stored in this solution or in this solution with $10 \% \mathrm{C} 10-2$ (see below) to reduce sticking of explants (M. Tessier-Lavigne, personal communication). Tissues that needed to be stored for $>1$ hr were kept at $4^{\circ} \mathrm{C}$ until plating.

Explants were cultured in collagen gels and overlaid with medium. The medium used is termed C10-2 media [85\% Eagle's MEM containing $0.6 \%$ glucose, 2 mM L-glutamine, $2 \%$ chick embryo extract (Life Technologies), and $10 \%$ fetal equine serum]. Collagen I was prepared from adult rat tails as recommended by Dr. M. Tessier-Lavigne, based on previously described methods (Ebendal, 1989). For $100 \mu \mathrm{l}$ of collagen gel we added $\sim 55 \mu \mathrm{l}$ of concentrated collagen to a solution containing $10 \mu \mathrm{l}$ of $10 \times$ MEM (Sigma, St. Louis, MO), $3 \mu$ l of $20 \%$ glucose, $1.9 \mu 1$ of 0.8 $\mathrm{M} \mathrm{NaHCO}_{3}, \mathrm{H}_{2} \mathrm{O}$, and growth factor (diluted to $50 \times$ in $\mathrm{C} 10-2$ media) to a total of $45 \mu \mathrm{l}$. BDNF (Sigma) and neurotrophin-3 (NT-3) (Sigma) were used at concentrations of $2-10 \mathrm{ng} / \mathrm{ml}, 2.5 \mathrm{~S}$ NGF (purified from male rat submaxillary glands, generously provided by Eugene Johnson, Washington University, St. Louis, MO) was used at 0.5-50 ng/ml. Cultures were grown on $22 \mathrm{~mm}$ coverglasses (number 1) in a total volume of 35-45 $\mu \mathrm{l}$ of collagen gel. A 15-20 $\mu$ l cushion of collagen was first spread out over approximately one-third of the surface of the coverglass. The collagen was permitted to polymerize for $20-30 \mathrm{~min}$ at $35^{\circ} \mathrm{C}$ in a humidified chamber. Explant pieces were then placed on top of the cushion in a drop of C10-2 media. The drop of liquid was aspirated, and the explants were overlaid with 20-25 $\mu$ l of collagen gel mixture. One to four trigeminal ganglion explants or one to three target-explant pairs were cultured on a single coverglass, separated by $\sim 3 \mathrm{~mm}$. The presence of more than one explant or explant pair on a single coverglass did not appear to affect explant outgrowth under the conditions that were used. Explants were separated by $277 \pm 88 \mu \mathrm{m}$ (SD) in experiments with E12 target explants $(n=182), 296 \pm 104 \mu \mathrm{m}$ in E13 cocultures $(n=41)$, and $265 \pm 92 \mu \mathrm{m}$ in experiments with E14 target explants $(n=80)$. After permitting gelation of the collagen mixture as described above, the gels were submerged in $250 \mu \mathrm{l}$ of $\mathrm{C} 10-2$ media containing growth factors as indicated. For function-blocking studies using anti-neuropilin-1 (generously provided by Dr. Marc Tessier-Lavigne), antibody was included in the top collagen and added to the media $(200 \mu \mathrm{l})$. In these experiments, three explant pairs were included in each dish, and observations were performed at $1.5 \mathrm{~d}$ in vitro.

Cultures were incubated at $35^{\circ} \mathrm{C}$ in a humidified environment containing $5 \% \mathrm{CO}_{2}$ and observed at $1 \mathrm{~d}$ intervals for up to $3 \mathrm{~d}$. Observations were performed on a Leitz Diavert microscope equipped with Hoffman Contrast optics. Digital images of explants were taken with a Sony DKC-5000 three-chip color CCD camera. Contrast enhancement and, in some cases, background subtraction were performed with Adobe Photoshop software.

Cultures were fixed by submerging the coverglass in $0.1 \mathrm{M}$ cacodylate, pH 7.4 , containing $5 \mathrm{mM} \mathrm{CaCl} 2$ and $4 \%$ paraformaldehyde $\left(35^{\circ} \mathrm{C}\right)$. The cultures were then transferred to a $4^{\circ} \mathrm{C}$ environment for $1.5 \mathrm{hr}$ and rinsed with $0.1 \mathrm{M}$ cacodylate buffer. In early experiments, cultures were stored in a PBS-blocking solution containing $1 \%$ Triton $\mathrm{X}-100,1 \%$ fish gelatin, $1 \%$ goat serum, $1 \%$ horse serum, and bovine serum albumin at a concentration of $1.4 \mathrm{mg} / \mathrm{ml}$. In later experiments, the Triton X-100 was replaced with $0.02 \%$ saponin to reduce extraction of membranes, and BSA was not included in the blocking buffer. Neurites were labeled with a monoclonal antibody mixture containing mouse anti-GAP-43 (Sigma) and mouse anti- $\beta$-tubulin III (Sigma). Coverglasses were rinsed in carrier solution, PBS block as described above but without Triton X-100, or, in early experiments, with $0.3 \%$ Triton $\mathrm{X}-100$. The antibodies were diluted in the carrier solution. A slightly higher concentration of antibodies was used for labeling collagen gels as compared with planar cultures because the gels had a volume of 35-40 $\mu \mathrm{l}$, and the coverglasses were overlain with a total of $120 \mu \mathrm{l}$ of antibody-containing solution. The labeling was performed overnight (12-16 hr), and the coverglasses were washed in carrier four or five times, $1.5-2 \mathrm{hr}$ each, at $4^{\circ} \mathrm{C}$ with agitation. A Cy3-conjugated donkey anti-mouse antibody (Jackson ImmunoResearch, West Grove, PA) diluted in carrier was used as the secondary antibody. Incubations and washes were performed as for the primary antibody. For observations, coverglasses were mounted in Vectashield (Vector Laboratories, Burlingame, CA) mounting medium on larger coverglasses or slides and sealed with nail polish. To prevent the gels from being compressed, slats prepared from coverglass or frame seal chambers (MJ Research, Inc.) were used to support the sides of the coverglasses. Observations were made on a Leitz (Wetzlar, Germany) Orthoplan microscope, but stored images were typically obtained on either of two confocal microscopes (Bio-Rad, Hercules, CA; or Zeiss, Oberkochen, Gemany). A series of image planes (Z-series) was obtained, and the projection of the series was stored. Color coding of depth was used to facilitate assessment of the portion of a target explant being approached by axons.

Whole-mount immunohistochemical labeling of peripheral nerves in rat embryos was performed as described by Kitsukawa et al. (1997). Monoclonal 2H3 anti-neurofilament was generously provided by Drs. Kim Lim and Douglas Engel. For development of the diaminobenzidine product, we followed the protocol of Ernfors et al. (1994). Anterograde labeling of mandibular nerves with 1,1'-dioctadecyl-3,3,3',3' -tetramethylindocarbacyanine perchlorate (DiI; Molecular Probes, Eugene, OR) was performed as described by Farbman and Mbiene (1991), except that labeling was completed within $7 \mathrm{~d}$, because embryos were kept at $35^{\circ} \mathrm{C}$ after placement of DiI crystals. Embryos were cleared with $80 \%$ glycerol and $20 \%$ PBS and imaged.

\section{RESULTS}

The lingual nerve develops between E13 and E14, after most major mandibular nerve trunks have formed. Mandibular afferents that will ultimately innervate medial structures appear to be restricted from medial targets initially. We used a whole-mount nerve-labeling method to study the development of mandibular nerve branches (cf. Hogg and Bryant, 1969; Lumsden, 1982).

Rat trigeminal ganglion neurons are born between E11 and E14 (Altman and Bayer, 1988). On E12, the developing mandible consists of two unfused arches that extend ventrally from the region of the posterior trigeminal ganglion (tg) (Fig. $1 C, I b)$ and then turn medially (Fig. $1 A, I b)$. The anterior portion of the tongue that will be innervated by the lingual nerve arises from the medial anterodorsal region of E12 arch Ib, facing the back of the embryo (Fig. 1B, dotted line). Pioneer growth cones destined for the mandible exit the ganglion on E12 (Fig. $1 C, m n$ ) and enter arch Ib.

By E13 the arches Ib have fused medially (Fig. $1 F$ ), and the dorsoanterior swelling that will become the tongue is evident (Fig. $1 F$, dotted line). Mandibular nerve axons $(m n)$ have advanced the length of the lateral aspect of the mandible (Fig. 1D) and turned medially (Fig. $1 E, F)$, remaining deep within the mesenchyme of arch Ib. We observed that the chorda tympani (Fig. $1 D, F, c t$ ) which carries prospective taste bud afferents of the geniculate ganglion, has already entered the presumptive tongue laterally. We believe this is the first demonstration that chorda tympani axons pioneer the innervation of the tongue. After the mandibular nerve has progressed approximately halfway through arch Ib ( $\sim$ E13.3), major branches of the mandibular nerve form 

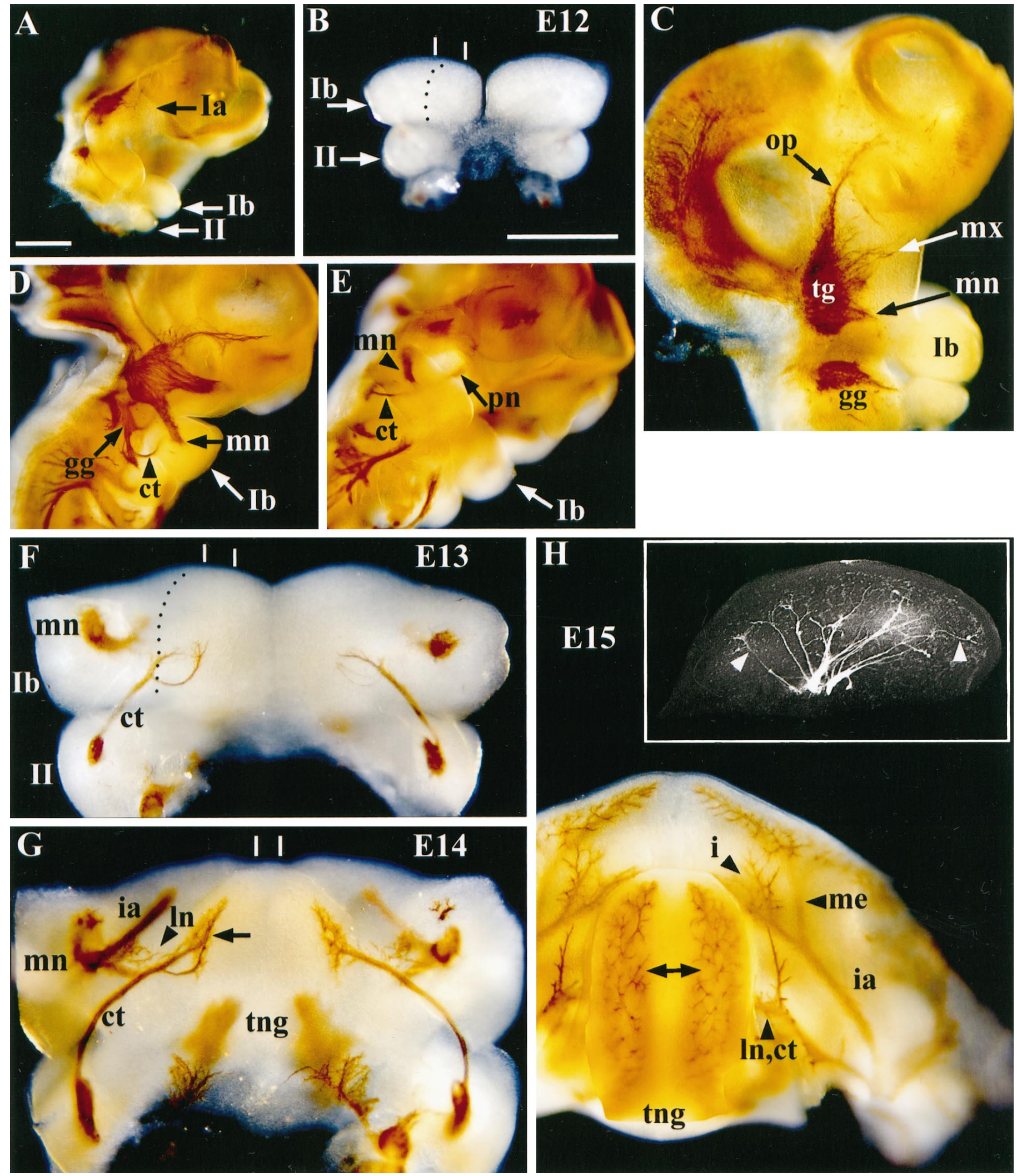

Figure 1. Development of rat mandibular innervation from E12 to E15. $A-C, \mathrm{E} 12 ; D-F, \mathrm{E} 13 ; G, \mathrm{E} 14 ; H$, E15. Concerning the mandibular arches, anterior is toward the top in $B$ and $C$ and $F-H$ and toward the top right corner of $A, D$, and $E$. Scale bars, $0.5 \mathrm{~mm}$. The scale bar in $A$ applies to $A$ and $C-E$; the scale bar in $B$ applies to $F-H$. E12: $A$, Anterior lateral view of the E12 rat head shows the full extent of the mandibular $(I b)$ and hyoid $(I I)$ arches. The trigeminal and geniculate ganglia can be seen, but are better appreciated in the lateral view $(C)$. $B$, Dorsal view of arches $I b$ and $I I$ reveals the region that will give rise to the tongue (dotted line). This surface is hidden in $A$ and $C$. Two vertical lines show the position of the parasagittal section of arch $\mathrm{Ib}$ used for medial explants. $C$, Lateral view of E12 rat head shows the location of the trigeminal $(\mathrm{tg})$ and geniculate ganglia ( $g g$ ) and the relative states of development of the opthalmic (op), maxillary $(m x)$, and mandibular $(m n)$ nerves. This embryo has slightly more advanced (Figure legend continues) 
(Fig. 1G). These major branches include the inferior alveolar (Fig. $1 G, i a$ ) and buccal (data not shown) branches. The lingual branch $(l n)$ does not begin to develop until late E13 or early E14. By E14, an immature tongue is apparent (Fig. 1G, tng), and because of nonuniform growth of cranial structures, the dorsal tongue surface is now roughly parallel to the whiskerpad. The first axons of the lingual nerve (Fig. $1 G, \ln$ ) have just entered the tongue laterally, toward its posterior. These pioneer lingual axons appear to first grow toward and then nearby the chorda tympani nerve (M. Rochlin and A. Farbman, unpublished observations). The majority of the axons in the tongue at early E14 (arrow) are derived from the geniculate ganglion via the chorda tympani nerve. This was confirmed by DiI staining, which revealed that only a few trigeminal axons had entered the tongue at early E14 (data not shown). On entering the tongue, the lingual nerve axons will turn anteriorly and then course through the lateral mesenchyme of the tongue.

Between E14 and E15, the tongue becomes thicker and narrower. Evidently, the lateral edges of the tongue at E14 fold down to become the sides of the tongue at E15, leading to the narrowing. As a consequence, whereas the chorda tympani and lingual afferents appeared to enter the tongue near its dorsal posterior surface, they now appear to enter the lateral surface of the tongue ventrally. Thus, the image of the right side of an E15 mandible (Fig. $1 H$ ) is a composite of multiple focal planes, including one for the ventral site of lingual nerve entry $(l n, c t)$ and another for the spray of sensory endings approaching the dorsal surface of the tongue (double-headed arrow). The inset shows lingual nerve axons (arrowheads) stained with DiI, indicating that a subset of the sensory fibers in the tongue at E15 are indeed derived from the trigeminal ganglion. Note that lingual afferents have progressed medially, but neither the lingual nerve afferents nor the chorda tympani afferents have completed their growth into the midline region of the tongue. Mental nerve afferents $(m e)$ that will ultimately innervate the chin are also restricted away from their medial targets at these stages. Despite the expansion of the lingual arbors between E14 and E15, unequivocal contact with the dorsal tongue epithelium is not observed until E16 (Farbman and Mbiene, 1991).

In summary, mandibular nerve axons remain tightly bundled and deep in arch Ib mesenchyme until mid-E13. The first mandibular nerve axons do not invade the tongue, but instead turn to grow parallel to it (Fig. $1 G, i a$ ). The lingual nerve develops late on E13, and its afferents arrive in the posterior tongue early on E14, after the arrival of chorda tympani axons. Although initially restricted to the lateral tongue, by E15, lingual afferents are evident beneath all but the most medial $\sim 25 \%$ of the tongue epithelium. Several questions are raised by these observations. Are early trigeminal axons attracted into the arch by target-derived chemoattractants? Are diff usible repellants responsible for the restriction of axons away from the tongue between E13 and E14 and away from the midline between E14 and E15? To address these questions, we assessed the influence of arch Ib or tongue explants on axon outgrowth from posterior trigeminal explants in the presence and absence of exogenous trophic factors. We used posterior trigeminal ganglion explants because this portion of the ganglion is enriched in neurons that ultimately innervate the tongue (Gregg and Dixon, 1973).

\section{Trigeminal ganglion explants depend on trophic support for axon outgrowth in collagen gels}

It has been established that random or whole trigeminal ganglion explants from mouse (Lumsden and Davies, 1983) and rat (Arumäe et al., 1993), when cultured in isolation, do not extend axons into collagen gels in the absence of added growth factors. Arumäe et al. (1993) (cf. Paves et al., 1994) have also demonstrated that BDNF, NT-3, and NT-4/5 (2-10 ng/ml) stimulated axon outgrowth from E12-E13 explants, whereas NGF was ineffective (even at $50 \mathrm{ng} / \mathrm{ml}$ ) on ganglia dissected from E12 embryos but stimulated outgrowth from ganglia dissected from E13 forward (for review, see Davies, 1997). Because it was not clear whether the previous work had included the posterior trigeminal ganglion, we examined the neurotrophin dependence of axon outgrowth from this portion of the ganglion. We confirmed that the earlier findings apply to posterior trigeminal ganglion. We found that E12-E14 posterior trigeminal ganglion explants do not extend axons in the absence of added growth factors (Fig. 2A, E12). At E12, NT-4/5 appeared to promote the most outgrowth (Fig. 2B), followed by BDNF and then NT-3. We also found that the potency of NT-3, NT-4/5, and to a lesser extent, BDNF, declined between E13 and E14, and that sensitivity to NGF developed in ganglia dissected from E12.5 embryos, indicating that this shift had been determined by E12.5 (data not shown).

\section{Presumptive target explants support trigeminal ganglion explant outgrowth}

We cocultured posterior trigeminal ganglion explants and arch Ib from E12-E13 embryos or dorsoanterior tongue explants from E14 embryos in the absence of added neurotrophins. The E12-E13 explants consisted of parasagittal sections taken from the region indicated in Figure $1 B, F$ (vertical lines), and E14 explants consisted of parasagittal sections of dorsal anterior tongue that excluded the septum. In E12 cocultures $(n=16)$ little outgrowth was promoted even after $2 \mathrm{~d}$ in vitro (Fig. $2 C$ ),

\section{$\leftarrow$}

nerve development than is typical at the stage of dissection and was selected to better delineate the three major nerves of the trigeminal ganglion. E13: $D, E$, By $\sim$ E13.3, the mandibular nerve has advanced ventrally $(D)$ and turned medially $(E)$. In $E$, because of the angle of the image, the palatal nerve ( $p n)$ appears to continue from the chorda tympani $(c t)$, but this is not the case. The palatal nerve [to the right of the arrow designating the geniculate ganglion $(g g)$ in $D$ ] arises from a position dorsal to the chorda tympani and does not combine with the chorda tympani. $F$, Dorsal view of arch $I b$ reveals the presumptive tongue surface (dotted line). Chorda tympani axons have already entered the tongue posteriorly near the dorsal surface, but no trigeminal axons could be detected until E14. The mandibular nerve axons are still mostly bundled deep in the mesenchyme, although the pioneers of the major nerves $(H)$ are beginning to segregate from the main nerve trunk. Most of the axons that have grown medially will contribute to the inferior alveolar $(i a, G, H)$ branch and turn parallel to the lateral border of the tongue. The medial E13 explants were taken from the area indicated by the vertical bars. E14: $G$, Immature tongue (tng) has formed. Chorda tympani afferents have turned away from their medial course to grow anteriorly or posteriorly along the lateral mesenchyme. Lingual nerve $(l n)$ afferents arising from the mandibular nerve have just begun to enter the tongue. Medial tongue explants were taken from the area indicated by the vertical bars. Note that E14 medial tongue explants are sagittal (centered around the midline), whereas E12-E13 medial explants are parasagittal. E15: $H$, Chorda tympani axons and lingual nerve axons combine (ln, $c t)$ and continue to infiltrate the tongue, but both sets of axons appear to be restricted away from the midline. The inferior alveolar ( $i a)$ and its major branches [the incisor $(i)$, and the mental nerve ( $m e$ )] have developed. Inset, Lingual nerve afferents labeled with DiI have entered the tongue and advanced toward the dorsal epithelium. 

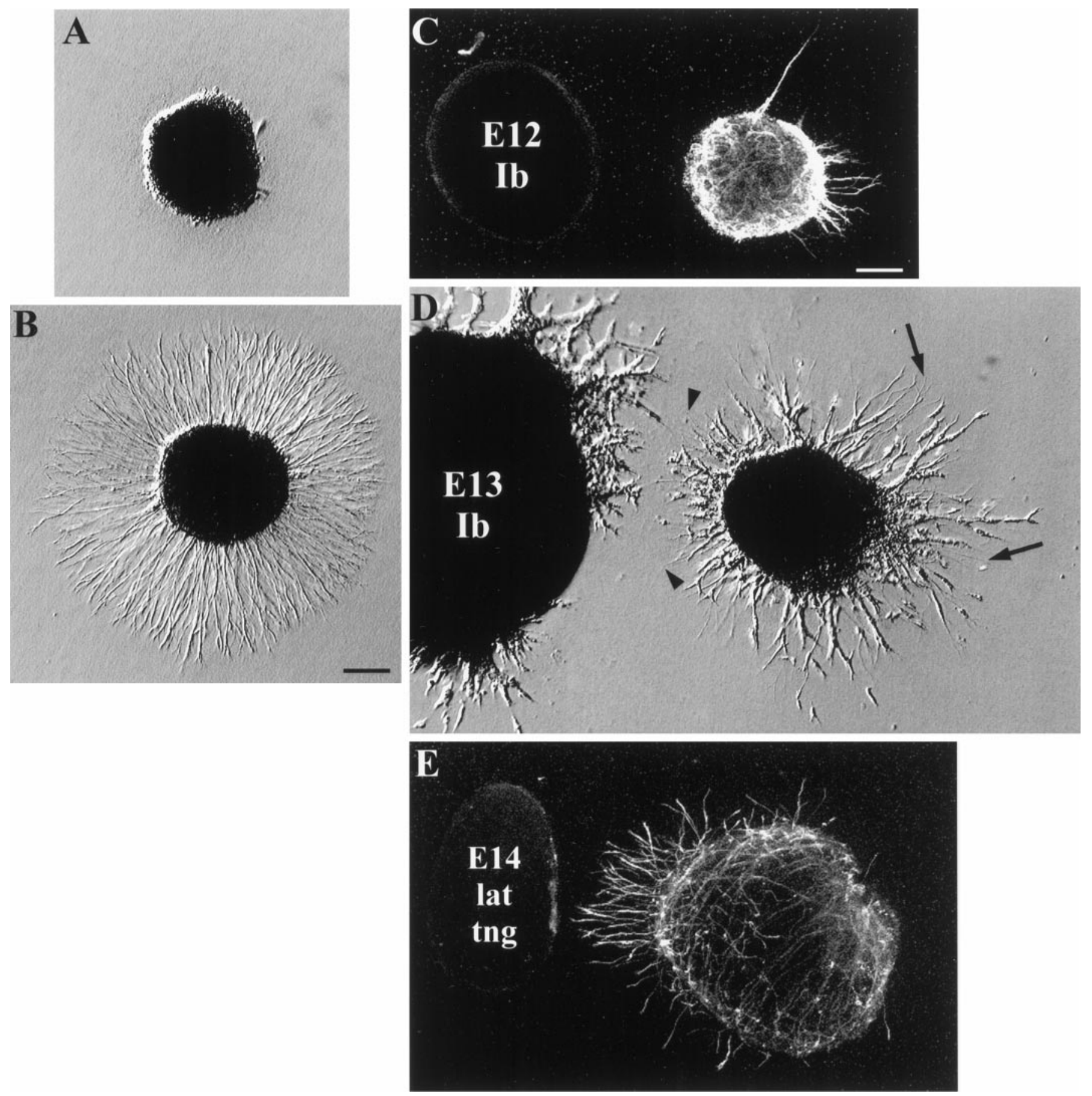

Figure 2. Trigeminal ganglion explants extend axons into collagen gels only if cultured with exogenous growth factors or presumptive target tissue. $A-D$, Cultures shown at $2 \mathrm{~d}$ in vitro; $E$, at $1 \mathrm{~d}$ in vitro. In the absence of target explants, no axons grew out from this E12 posterior trigeminal ganglion explant $(A)$. In the presence of $5 \mathrm{ng} / \mathrm{ml} \mathrm{NT}-4 / 5$, a uniform halo of outgrowth is observed $(B)$. Presumptive mandibular targets secrete trophic factors that promote axon outgrowth at E12 $(C), \mathrm{E} 13(D)$, and E14 $(E)$. There is no outgrowth toward the target explant at E12. At E13, there is more outgrowth toward the target explant after $2 \mathrm{~d}$ (arrowheads) than seen at E12, but outgrowth on the distal side of the explant (arrows) is longer. At E14, explants of dorsoanterior tongue excluding the midline apparently attract axon outgrowth. In $C$ and $E$, cultures were fixed, stained for $\beta$-tubulin III and GAP-43, and imaged with a confocal microscope (see Materials and Methods). Scale bar, $100 \mu \mathrm{m}$.

but this was greater than that observed from trigeminal explants cultured alone. E13 arch Ib explants (Fig. 2D, $n=10$ ) stimulated more axon outgrowth than E12 explants. The number of axons appeared to increase more rapidly between 2 and $3 \mathrm{~d}$ in vitro than between 1 and $2 \mathrm{~d}$. The Hoffman contrast image shows that non-neuronal cells proliferate and accom- pany axon outgrowth stimulated by the target explant. E14 dorsoanterior tongue explants stimulated rapid, although sparse, outgrowth from trigeminal explants (Fig. $2 E, n=21$ ). Thus, as was reported for mouse trigeminal targets (Lumsden and Davies, 1983), presumptive trigeminal targets secrete trophic factors that support axon outgrowth. 


\section{Presumptive target explants derived from E12 embryos repel trigeminal ganglion axon outgrowth}

Surprisingly, axons grew away from arch Ib explants dissected from E12 rat embryos (Fig. $2 C$ ), suggesting that, in addition to trophic factors, a repellant (or group of repellants) was also being secreted from the presumptive target. In 13 of 16 cocultures, outgrowth was longer in the quadrant opposite the arch Ib explant than in the quadrant facing the arch Ib explant. To enhance the outgrowth so as to enable quantitation of the repellant influence, we included $5 \mathrm{ng} / \mathrm{ml}$ doses of the following growth factors: BDNF, NT-3, BDNF + NT-3, and NT-4/5. Regardless of which factors were used, explant outgrowth was repelled, and the greater numbers of axons made the phenomenon more apparent (Fig. $3 A$ ) and quantifiable (Table 1). In the presence of growth factors the repellant influence was evident after $1 \mathrm{~d}$ in vitro, and the difference in axonal outgrowth in proximal and distal quadrants was significant in all cases ( $p \leq 0.01$; data not shown). NT-4/5-stimulated axon outgrowth appeared to be more strongly repelled than outgrowth stimulated by the other factors, but the difference was subtle. Both BDNF and NT-4/5 stimulated more rapid axon extension than NT-3 (Table 1). Axons did not grow toward arch Ib explants after $2 \mathrm{~d}$ in vitro. This could be attributable to either maintenance of the repellant influence or the fact that once axons are induced to grow away from the arch, their growth cones advance out of the range of subsequently produced attractants.

Logically, the target cannot repel the axons that will innervate it in vivo, so we considered alternative interpretations. Were we inadvertently selecting for axons destined for alternative trigeminal targets, such as the whiskerpad, that might reasonably be repelled by the developing tongue? We found that E12 arch Ia, the developing whiskerpad, was also repellant (Table 1). Furthermore, repulsion was observed regardless of whether we used the posterior or anterior trigeminal ganglion with arch Ib (Table 1), consistent with the possibility that all axons produced by E12 trigeminal ganglia are repelled by these two presumptive targets. Given that axon outgrowth stimulated by all of the known effective neurotrophins was repelled, and also that the outgrowth stimulated by arch Ib without added growth factors was repelled, it is unlikely that we have selected for axons destined for an alternative peripheral target. Although we cannot eliminate the possibility that we have selected for the CNS branch, it seems unlikely that the distal portion of arch Ib would be important in such a remote phenomenon. Furthermore, at least in chick, both branches appear to express a receptor for the most likely candidate for the chemorepellant (see below) (Kobayashi et al., 1997). These findings suggest that the appropriate axons are repelled by the presumptive target explant.

Is the repellant influence derived from a portion of the presumptive target explant that does not become innervated by the axons? Because the majority of trigeminal axons will ultimately innervate epithelial- and subepithelial-derived tissue, we tested whether it was the epithelium or the central mesenchyme that produced the repellant. By peeling the epithelium from the arch explant and placing it and the mesenchyme on opposite sides of the trigeminal ganglion explant, we found that the epithelium is the source of the repellant, not the central mesenchyme (Fig. 3D). We eliminated the unlikely possibility that the mesenchyme exerts a repellant influence that is much weaker than that of the epithelium by coculturing trigeminal ganglion explants with mesenchyme and without the epithelium. Under these conditions the mesenchyme did not repel axon outgrowth $(n=3$, data not shown). However, the most peripheral band of mesenchymal cells may be damaged by the separation procedure, so we cannot exclude the possibility that they too produce repellant.

Trigeminal axons do not innervate the ventral epithelium of arch Ib, so we tested whether this might be the exclusive source of the repellant. Arch Ib explants were cut into dorsal, ventral, anterior, or posterior halves and cocultured with posterior trigeminal explants. All halves were repellant, and the anterior and ventral halves appeared to be more repellant than the dorsal and posterior halves (data not shown). Given that the dorsal and posterior half explants would contain anterior and ventral epithelium, respectively, we used a diagonal cut to generate ventroanterior and dorsoposterior half explants (Fig. 3E). The ventroanterior half, as expected, was strongly repellant (Fig. 3B), but the dorsoposterior half was not repellant (Fig. $3 C$ ). These data imply that the ventral surface, which does not get innervated, is repellant (Fig. 3E, top panel, dark surface). Because the dorsal half was repellant, but not the posterodorsal half, these data also establish that the anterior quarter of the dorsal surface is repellant (Fig. $3 E$, bottom panel, dark surface). The anterodorsal surface gives rise to the anterior portion of the tongue, which is innervated by the lingual and chorda tympani nerves.

To determine how far laterally the repellant was distributed, parasagittal ventral anterior arch Ib explants from the center or lateral aspect of the arch were tested. Central arch Ib also exerted a net repellant influence, albeit weaker than that of medial explants (Table 1). The lateral third of arch Ib was repellant in only four of eight cases. Presumably one edge of these explants was repellant and only faced the ganglion explant in half of the trials. Thus, the medial two-thirds of each arch are repellant (Fig. 3E, dark surfaces), but not the lateral third.

\section{Neuropilin-1 is required for the repulsive influence of arch Ib}

Near the completion of our study, knock-outs of semaphorin III/D or its putative receptor neuropilin-1 were shown to cause severe disruption of mandibular nerve development (Kitsukawa et al., 1997; Taniguchi et al., 1997). We therefore tested whether this system was required for arch Ib to repel posterior trigeminal ganglion axons. Neutralizing anti-neuropilin-1 blocked the repellant influence of E12 arch Ib in the presence of NT-4/5 (Fig. $3 F$ ) or in its absence (Fig. 3G). In the absence of added growth factors, there was more outgrowth toward arch Ib explants than away, consistent with the possibility that trophic and, possibly, tropic factors are secreted by arch Ib (Fig. 3G, Table 1). Clearly, presumptive target tissue secretes multiple diffusible factors that differentially regulate axon extension and the direction of axon extension. Taken together, however, these data show that future targets of trigeminal ganglion neurons, such as the presumptive anterodorsal surface of the tongue, have a net repellant influence on these afferents. Ultimately, the target must not repel the axons that innervate it, so we tested the influence of presumptive target explants dissected from more mature embryos.

\section{E13 arch Ib explants repel axons during the first day in vitro but subsequently permit or attract trigeminal axon outgrowth toward the arch explant}

At E13, growth factor-supported axon outgrowth from posterior trigeminal ganglion explants was clearly repelled during the first day of outgrowth (Fig. 4A, Table 1), but from the second day in vitro forward, axons grew toward the presumptive target explant (Fig. 4B). The repellant influence was found to be present as 

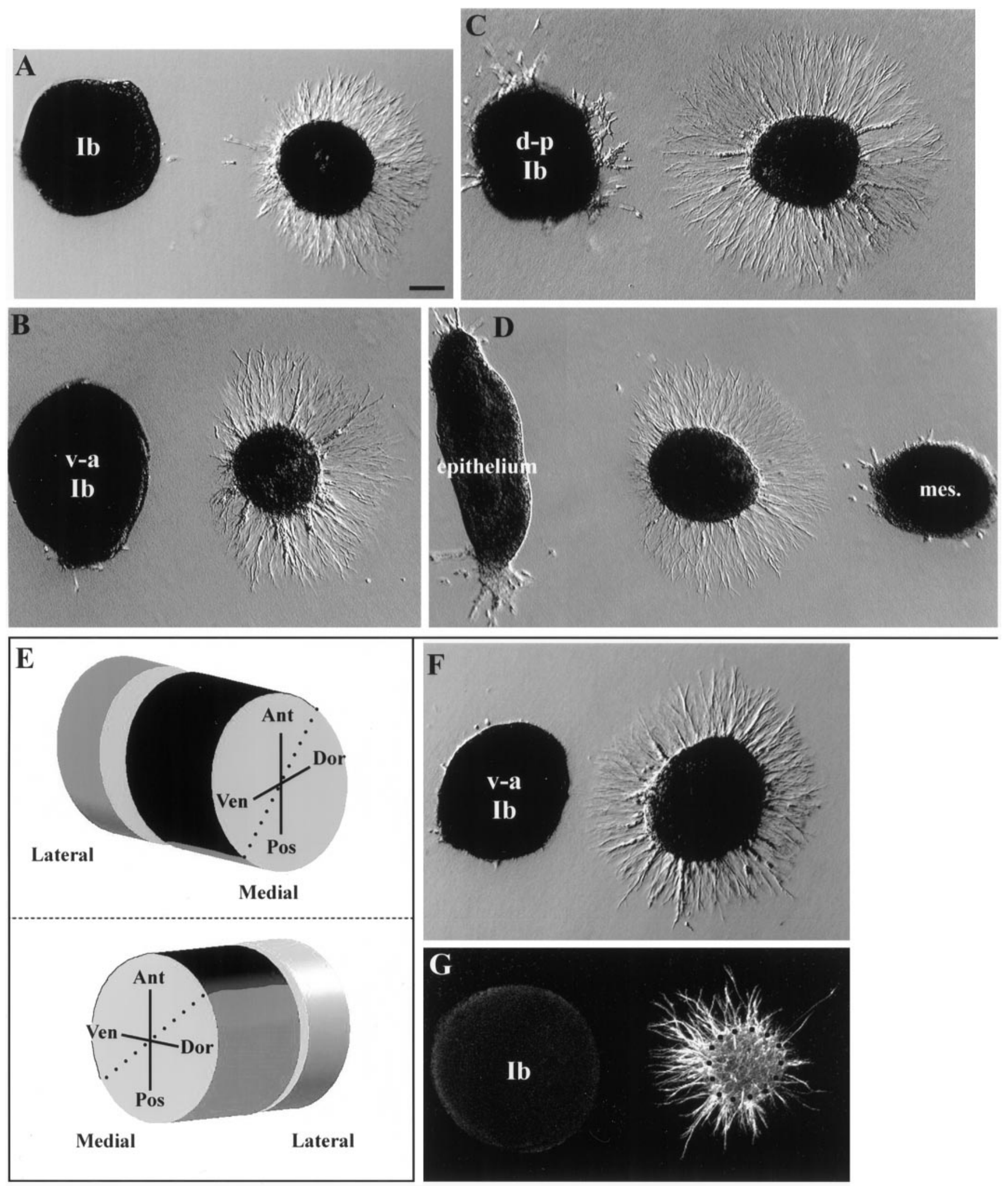

Figure 3. Arch Ib has a net repellant influence on growth factor-stimulated axon outgrowth from E12 posterior trigeminal ganglion explants; this influence is not uniformly distributed throughout the arch, and it depends on functional neuropilin-1. $A-D$, Distribution of the repellant activity. NT-4/5 was used to stimulate outgrowth, and images were obtained after $2 \mathrm{~d}$ in vitro. A, This parasagittal explant of arch Ib repels axon outgrowth. To determine the distribution of repellant within the arch, the parasagittal disks were halved along one of three axes (dorsal-ventral, anterior-posterior, or anterior ventral-posterior dorsal; see $E$ ). The repellant influence is secreted by anterior-ventral hemiexplants $(B)$ and all other half explants (data not shown) with the exception of the dorsal-posterior $(d-p)$ hemiexplants $(C)$. We also found that the repellant influence derived (Figure legend continues) 
lateral as the lateral edge of the presumptive tongue (Table 1). In some cases, cultures were stained for neurites with a mixture of anti-GAP-43 and anti- $\beta$-tubulin III and imaged with a confocal microscope. By color coding the image planes, we show that axons were able to grow toward the anterior surface of the explant. In this experiment, the anterior surface was positioned closest to the coverglass (red-yellow axons are close to the coverglass, blue are closer to the media-facing surface of the collagen gel). Initial experiments were performed with $5 \mathrm{ng} / \mathrm{ml}$ growth factor. This concentration of growth factor stimulated only a small halo of outgrowth by 24-29 hr in vitro. To increase the outgrowth during the first day of culture and more clearly visualize the repellant phenomenon, we used $10 \mathrm{ng} / \mathrm{ml}$ of these growth factors. With the lower concentrations of growth factors or no growth factors, outgrowth toward the target explant on the second and third day in vitro was more likely to reverse the bias from "away" to "toward" the target explant. Presumably, the higher growth factor concentration induced axonogenesis more efficiently, resulting in a higher percentage of neurons with axons that were turned away from the target explant during the first day in vitro. As was noted with ganglion explants derived from E12 embryos, NT-4/5 appeared to stimulate denser outgrowth than BDNF and NT-3. We tried to evaluate whether NGF-stimulated axon outgrowth was also repelled by E13 arch Ib, but this outgrowth is too short at $1 \mathrm{~d}$ in vitro to assess this (data not shown). Apparently, although NGF sensitivity is determined as early as E12.5, it is not expressed until late E13-early E14. However, heterochronic experiments discussed below suggest that this NGF-sensitive outgrowth is also repellant-sensitive.

These data suggest that tissue explants containing future targets of the trigeminal ganglion continue to exert a net repellant influence on their afferents from E13 to E14, independent of which neurotrophin is used to stimulate outgrowth. During this period, the earliest mandibular nerve axons turn away from their medial path to form the inferior alveolar nerves and its major branches, the mental, incisor, and molar nerves (Lumsden, 1982). Lingual afferents do not begin to grow toward the tongue until late E13 or early E14. Both of these observations are consistent with a physiological role for the repellant activity we describe.

\section{E14 lateral dorsoanterior tongue explants attract axon outgrowth from posterior trigeminal ganglion explants, but the midline remains repellant}

By E14, a structure that obviously resembles a mature tongue is apparent on the anterior dorsomedial surface of the former arch Ib (Fig. 1G). Lingual nerve axons have just begun to enter the tongue, and during the subsequent $24 \mathrm{hr}$ will grow radially toward the lateral region of the anterodorsal tongue surface (Fig. $1 H$ ). Do diff usible factors contribute to the development of this distribution pattern? To examine this, we conducted competition experiments in which medial and lateral explants derived from the same tongue were placed on opposite sides of posterior trigeminal ganglion explants, and the density of axon outgrowth on each side of the trigeminal ganglion explant was ranked (Table 1). In the absence of added growth factors, outgrowth was biased away from the medial tongue explant and toward the lateral tongue explant (Fig. 5A). Also, in cocultures of posterior trigeminal ganglion explants and either medial or lateral tongue, outgrowth was biased toward lateral tongue explants (Fig. $2 E$ ) but not toward medial explants (data not shown). These findings suggest that the attractive influence of the dorsal tongue surface is exclusively lateral at E14. Note that the axon outgrowth density was greater in the presence of both medial and lateral explants (Table 1, rank 5) than in the presence of the lateral explant alone (Table 1, rank 3; also compare Figs. $2 E, 5 A$ ). Presumably, both medial and lateral explants provide trophic support for outgrowth. We did not observe the exclusive growth toward target explants reported by Lumsden and Davies (1983, 1986), but axons occasionally took curvilinear paths to grow toward tongue explants (data not shown). The dorsoanterior tongue represents only a subset of the mandibular targets of the posterior trigeminal ganglion, but the tongue mesenchyme would be expected to trophically support (stimulate axonogenesis from) neurons destined for the ventral epithelium of the tongue (which might be repelled by the dorsal epithelium) or other mandibular targets. Our observations are consistent with the lateral tongue explants secreting both chemotropic and chemotrophic factors for posterior trigeminal ganglion axons.

To test for a diffusible repellant influence, we stimulated axon outgrowth with $2 \mathrm{ng} / \mathrm{ml}$ NGF. Under these conditions, axon outgrowth was repelled by medial tongue explants but not by lateral tongue explants (Fig. $5 B$, Table 1). Trigeminal ganglion explant outgrowth in the presence of either the medial (Table 1) or the lateral tongue explants confirmed this interpretation. This is consistent with a role for a diffusible repellant influence in restricting the initial outgrowth of lingual afferents to the lateral portions of the dorsal membrane. Thus, the first lingual nerve axons that enter the tongue appear to be attracted to its lateral surface and repelled by its medial surface.

These data indicate that the presumptive epithelial and subepithelial targets of lingual nerve axons ultimately exert a net attractive influence on the axons that innervate them, but not until those axons have used other means to navigate to the vicinity of the target from the ganglion.

\section{The repellant influence is downregulated between E12 and E14, not responsiveness to the repellant}

To ascertain whether the loss of the repellant influence of the lateral tongue is attributable to downregulation of the repellant or downregulation of responsiveness to the repellant, we cocultured heterochronic explants. Parasagittal explants of E14 dorsal tongue did not repel or bias NT-4/5-supported outgrowth from E12 posterior trigeminal ganglion explants (Fig. 5C), suggesting

\section{$\longleftarrow$}

from the epithelium, not the mesenchyme (mes.) of arch $\mathrm{Ib}(D)$. Although the epithelium begins as only a sheet of cells, it grows in vitro, whereas the mesenchyme contracts, leading to the apparent size difference in these two explants. E. The distribution of repellant within arch Ib. Top panel, Three-quarter view from the ventromedial perspective of an idealized right arch Ib. The repellant portion of the epithelium is indicated by the dark surface region. The most lateral third of arch Ib did not exert a net repellant influence. Bottom panel, Idealized arch Ib viewed from the dorsomedial perspective. The anterior quarter of the dorsal half has a net repellant influence, indicated in black. $F, G$, Neuropilin-1 is required for repulsion of trigeminal afferents. Cultures were imaged after $1.5 \mathrm{~d}$ in vitro. $F$, Neutralizing anti-neuropilin-1 blocks the repellant influence of E12 ventral and anterior $(v-a)$ arch Ib in the presence of NT-4/5. Compare with $A$. $G$, In the absence of added growth factors, blocking neuropilin-1 uncovers an attractive influence of entire parasagittal sections of E12 arch Ib. At least part of this influence is attributable to trophic factors secreted from arch Ib. Scale bar, $100 \mu \mathrm{m}$. 
Table 1. Trigeminal axon outgrowth bias in the presence of presumptive target explants

\begin{tabular}{|c|c|c|c|c|c|}
\hline \multirow[b]{2}{*}{ Explants } & \multirow{2}{*}{$\begin{array}{l}\text { Growth factor } \\
(\mathrm{ng} / \mathrm{ml})\end{array}$} & \multicolumn{2}{|c|}{ Mean axon outgrowth \pm SEM } & \multirow[b]{2}{*}{$\mathrm{N}$} & \multirow[b]{2}{*}{$p$ value $<$} \\
\hline & & Proximal quadrant & Distal quadrant & & \\
\hline \multicolumn{6}{|l|}{ E12 } \\
\hline \multirow{4}{*}{$\begin{array}{l}\text { Medial arch Ib vs } \\
\text { posterior trigeminal }\end{array}$} & BDNF (5) & $74 \pm 11$ & $165 \pm 22$ & 9 & 0.001 \\
\hline & NT-3 (5) & $33 \pm 11$ & $96 \pm 16$ & 8 & 0.0001 \\
\hline & NT-4/5 (5) & $92 \pm 10$ & $182 \pm 9$ & 24 & 0.0001 \\
\hline & $\mathrm{BDNF}+\mathrm{NT}-3(5)$ & $64 \pm 8$ & $182 \pm 15$ & 8 & 0.0003 \\
\hline $\begin{array}{l}\text { Central arch } \mathrm{Ib} \text { vs } \\
\text { posterior trigeminal }\end{array}$ & NT-4/5 (5) & $146 \pm 17$ & $224 \pm 6$ & 14 & 0.0008 \\
\hline Epithelium/mesenchyme vs & NT-4/5 (5) & To epithelium & To mesenchyme & & \\
\hline posterior trigeminal & & $116 \pm 21$ & $257 \pm 14$ & 9 & 0.0001 \\
\hline $\begin{array}{l}\text { Ant/Ven arch } \mathrm{Ib} \text { vs } \\
\text { posterior trigeminal }\end{array}$ & NT-4/5 (5) & $122 \pm 19$ & $222 \pm 11$ & 10 & 0.0006 \\
\hline $\begin{array}{l}\text { Pos/Dor arch Ib vs } \\
\text { posterior trigeminal }\end{array}$ & NT-4/5 (5) & $223 \pm 13$ & $228 \pm 8$ & 14 & NS \\
\hline $\begin{array}{l}\text { Arch Ib vs posterior } \\
\text { trigeminal }\end{array}$ & NT-4/5 (5) & $63 \pm 12$ & $158 \pm 16$ & 7 & 0.006 \\
\hline $\begin{array}{l}\text { Arch Ia vs posterior } \\
\text { trigeminal }\end{array}$ & NT-4/5 (5) & $109 \pm 21$ & $194 \pm 15$ & 7 & 0.008 \\
\hline \multicolumn{6}{|l|}{ Anti-NP-1 } \\
\hline $\begin{array}{l}\text { Ant/Ven } \mathrm{Ib} \text { vs posterior } \\
\text { trigeminal }\end{array}$ & NT-4/5 (5) & $213 \pm 9$ & $203 \pm 9$ & 12 & NS \\
\hline Ib vs posterior trigeminal & -GFs & $7 \pm 1$ & $2 \pm 1$ & 6 & 0.001 \\
\hline \multicolumn{6}{|l|}{ E13 } \\
\hline \multirow{3}{*}{$\begin{array}{l}\text { Medial arch } \mathrm{Ib} \text { vs } \\
\text { posterior trigeminal }\end{array}$} & BDNF (5-10) & $60 \pm 14$ & $191 \pm 26$ & 9 & 0.0001 \\
\hline & NT-3 (5-10) & $46 \pm 9$ & $92 \pm 6$ & 10 & 0.0001 \\
\hline & NT-4/5 (10) & $40 \pm 7$ & $139 \pm 15$ & 8 & 0.0001 \\
\hline $\begin{array}{l}\text { Central arch Ib vs } \\
\text { posterior trigeminal }\end{array}$ & BDNF (10) & $173 \pm 28$ & $311 \pm 16$ & 7 & 0.0022 \\
\hline \multicolumn{6}{|l|}{ E14 } \\
\hline \multirow{2}{*}{$\begin{array}{l}\text { Lateral tongue vs } \\
\text { posterior trigeminal }\end{array}$} & -GFs Lat only & $3 \pm 0$ & $1 \pm 0$ & 21 & 0.0001 \\
\hline & -GFs vs Med & $5 \pm 1$ & $1 \pm 0$ & 9 & 0.0001 \\
\hline \multirow{2}{*}{$\begin{array}{l}\text { Medial tongue vs } \\
\text { posterior trigeminal }\end{array}$} & NGF (2) Med only & $39 \pm 7$ & $169 \pm 8$ & 11 & 0.0001 \\
\hline & NGF (2) vs Lat & $27 \pm 13$ & $159 \pm 9$ & 9 & 0.0001 \\
\hline \multicolumn{6}{|l|}{ Heterochronics } \\
\hline \multirow{3}{*}{$\begin{array}{l}\mathrm{E} 12 \text { arch } \mathrm{Ib} \text { vs E14 } \\
\text { posterior trigeminal }\end{array}$} & NGF (1-2) & $32 \pm 12$ & $255 \pm 11$ & 12 & 0.0001 \\
\hline & NT-3 (25) & $33 \pm 16$ & $171 \pm 34$ & 8 & 0.003 \\
\hline & BDNF (10) & $11 \pm 11$ & $135 \pm 17$ & 5 & 0.003 \\
\hline $\begin{array}{l}\text { E14 lateral tongue vs E12 } \\
\text { posterior trigeminal }\end{array}$ & NT-4/5 (5) & $139 \pm 27$ & $136 \pm 25$ & 12 & NS \\
\hline
\end{tabular}

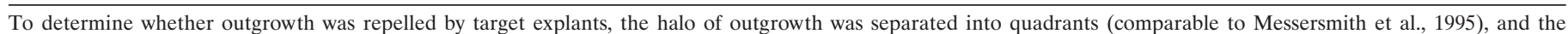

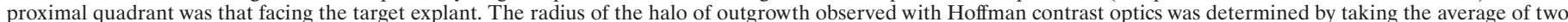

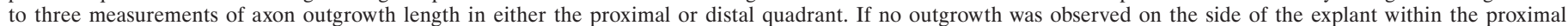

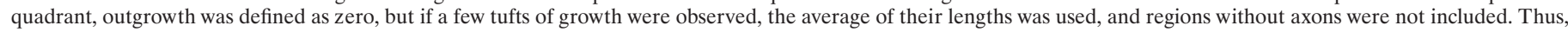

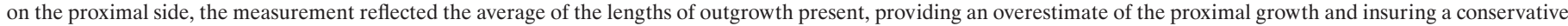

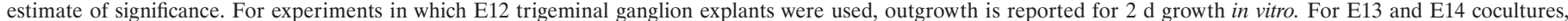

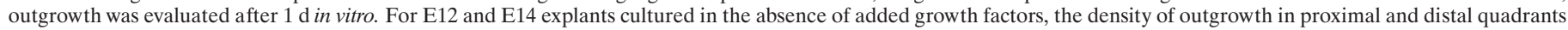

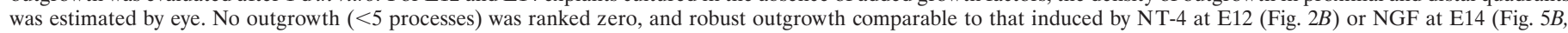

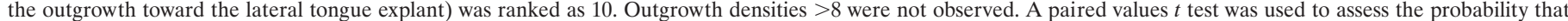

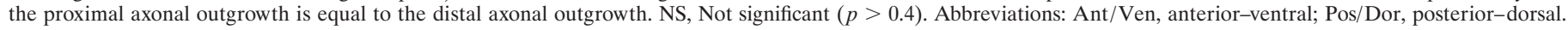

that the repellant influence had been downregulated. Conversely, E12 arch Ib was effective at repelling NGF-, NT-3-, and BDNFsupported outgrowth from E14 posterior trigeminal ganglion explants after 1-2 d in vitro (Fig. 5D, Table 1). NT-4 had decreased dramatically in potency by E14 and was not evaluated. Thus, E14 posterior trigeminal ganglion neurons are still sensitive to the repellant(s) secreted at E12 regardless of which of the most effective trophic factors are used to stimulate this outgrowth.

\section{DISCUSSION}

We report the first evidence of a net chemorepellant influence derived from a presumptive peripheral target. This finding argues 

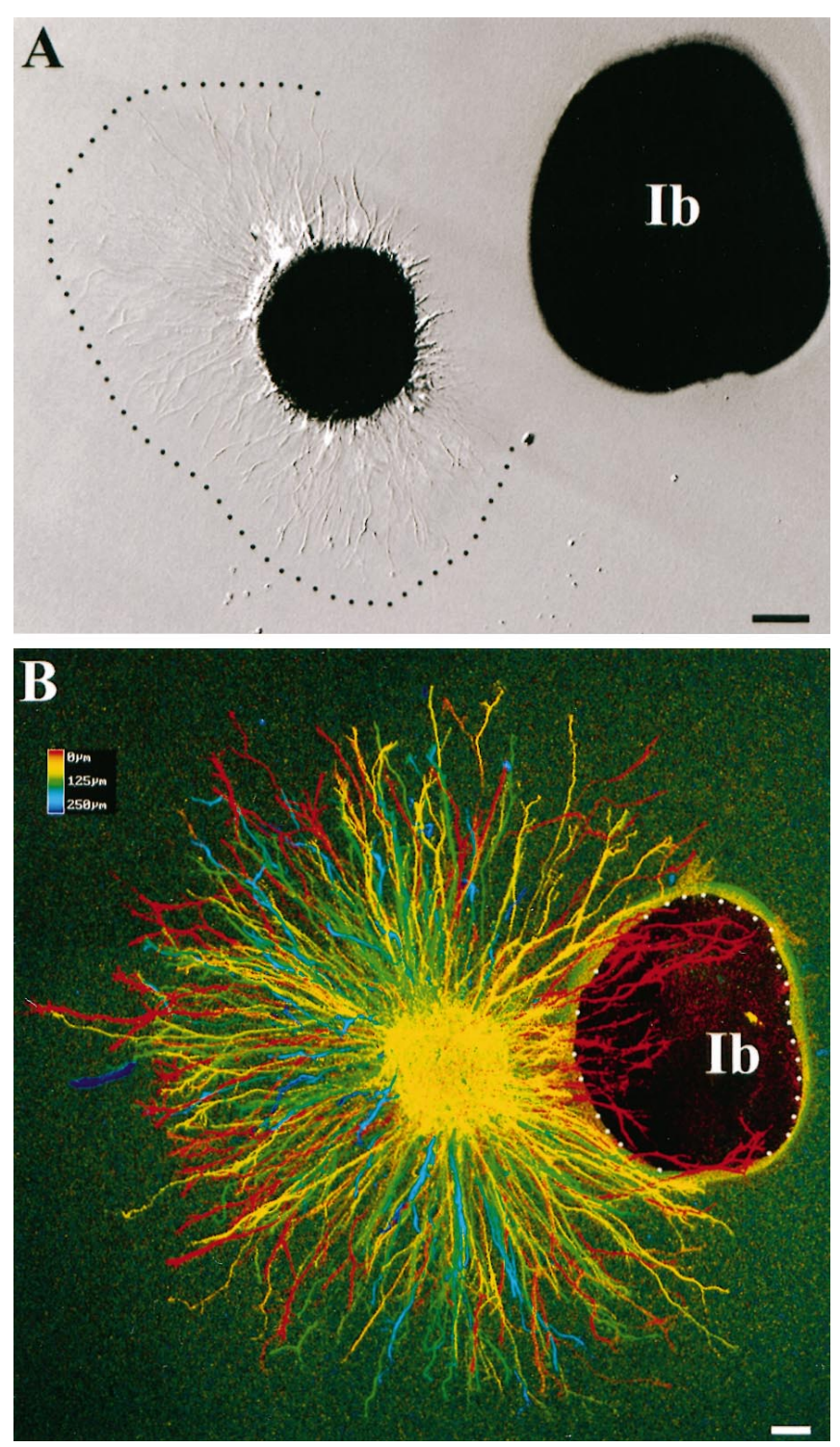

Figure 4. E13 arch Ib explants first repel and then permit or attract axon outgrowth from posterior trigeminal ganglion explants. $A$, During the first day in vitro, axons that have exited the trigeminal explant on the side facing arch Ib turn sharply away from it, whereas a large halo of growth is present (demarcated by black dots) on the side opposite the presumptive target. $B$, Same culture as in $A$, fixed at $3 \mathrm{~d}$ in vitro. By $3 \mathrm{~d}$ in vitro, axons have grown toward and around arch $\mathrm{Ib}$. The image is of a Z-series projection of $\mathrm{Cy} 3$ fluorescently labeled axons (see Materials and Methods) in which image planes have been color-coded for depth. Red staining encodes planes close to the substratum; yellow, green, and blue represent increasingly distal planes. In this case, we had positioned the explant anterior side down, so the presence of red axons indicates that axons have grown toward the anterior surface epithelium. Because of the high fluorescence background of the explant tissue and the low signal-to-noise ratio obtained with a $10 \times$ nonimmersion objective, we eliminated intermediate and low intensities within the perimeter of the area demarcated with white dots. This did not alter the pattern of axon staining. Note that $C$ is shown at a lower magnification than $B$. Scale bars, $100 \mu \mathrm{m}$.

against the long-standing assertion that target-derived chemoattraction is predominantly responsible for guiding growth cones from the trigeminal ganglion to its targets (Lumsden and Davies, 1983, 1986; Taniguchi et al., 1997). A repellant relationship has also been observed in the mouse (R. O'Connor and M. Tessier-
Lavigne, personal communication). The repellant phenomenon may not have been detected previously in mouse (Lumsden and Davies, 1983, 1986), because outgrowth was evaluated after $2 \mathrm{~d}$ in vitro. In the mouse, only $1.5 \mathrm{~d}$ elapsed between the exit of pioneer axons from the trigeminal ganglion and contact with the target epithelium (Davies et al., 1981; Stainier and Gilbert, 1990, 1991). If the dissections were performed after the presumptive target had become determined to downregulate its chemorepellant, then the $2 \mathrm{~d}$ interval would have been sufficient to allow for the transition from net repellant to net attractant influence in mouse. Without exogenous growth factors, little outgrowth would have been observed before the switch. Our data support Lumsden and Davies' (1983, 1986) reports of a chemoattractant influence of target epithelia, albeit at a later stage of development than they proposed. Our work complements recent findings of peripherally distributed repellant molecules and leads to a model of repellant function that suggests roles in turning and in the timing of nerve branch formation and target innervation that parallel hypothesized roles for chemorepellants in the CNS.

The most likely candidate for the repellant is semaphorin III/D. Semaphorin III/D knock-outs exhibit aberrant trigeminal axon trajectories consistent with loss of a significant repellant influence (Taniguchi et al., 1997). Semaphorin III/D has been shown to repel or collapse sensory axons (Luo et al., 1993; Fan and Raper, 1995; Messersmith et al., 1995), and both the central and peripheral branches of embryonic chick and mouse trigeminal ganglion axons express neuropilin-1, the semaphorin III/D receptor (Kobayashi et al., 1997; Kolodkin et al., 1997; cf. He and Tessier-Lavigne, 1997). Semaphorin III/D is diffusible (Luo et al., 1993; Püschel et al., 1996), and it is expressed by branchial arch epithelium (Wright et al., 1995; Adams et al., 1996; Giger et al., 1996; cf. Taniguchi et al., 1997). These data indicate that the semaphorin III/D-neuropilin-1 system is present in the branchial arches and their afferents and is necessary for normal pathfinding. Our data extend these observations by demonstrating that the dominant diff usible influence of the developing medial mandible is repellant and that neuropilin- 1 is responsible for mediating this repellant influence. Other diffusible chemorepellants are also present in the developing mandible. Netrin-1 (Serafini et al., 1994; Colamarino and Tessier-Lavigne, 1995; Varela-Echvarria et al., 1997) is expressed by tongue epithelium (Livesey and Hunt, 1997), and the netrin receptor deleted in colorectal cancer is present in sensory ganglia before E16 (Keino-Masu et al., 1996; Leonardo et al., 1997), but netrin does not repel sensory axons (Keynes et al., 1997). Neurotrophins such as BDNF and NT-4/5 have been reported to cause collapse of NT-3 trigeminal ganglion growth cones (Paves et al., 1994). However, BDNF- and NT-4/ 5 -supported growth cones were not collapsed by heterologous neurotrophins but were repelled by arch Ib. Furthermore, regardless of whether exogenous NT-3 and BDNF were used separately or together, trigeminal ganglion outgrowth was enhanced compared with the absence of growth factors, and the outgrowth was repelled by the arch.

What are the functions of the repellant influence? We localized the repellant influence to the epithelium, which presumably generates a radial gradient of repellant that is strongest near the epithelium and weakest in the core mesenchyme. Our work suggests three distinct functions for this repellant influence. First, the repellant may restrict axons away from nontarget regions of target-containing tissue. The ventral epithelium, which does not become innervated by trigeminal afferents, is repellant, consistent with such a role. Second, because both the ventral and dorsal 

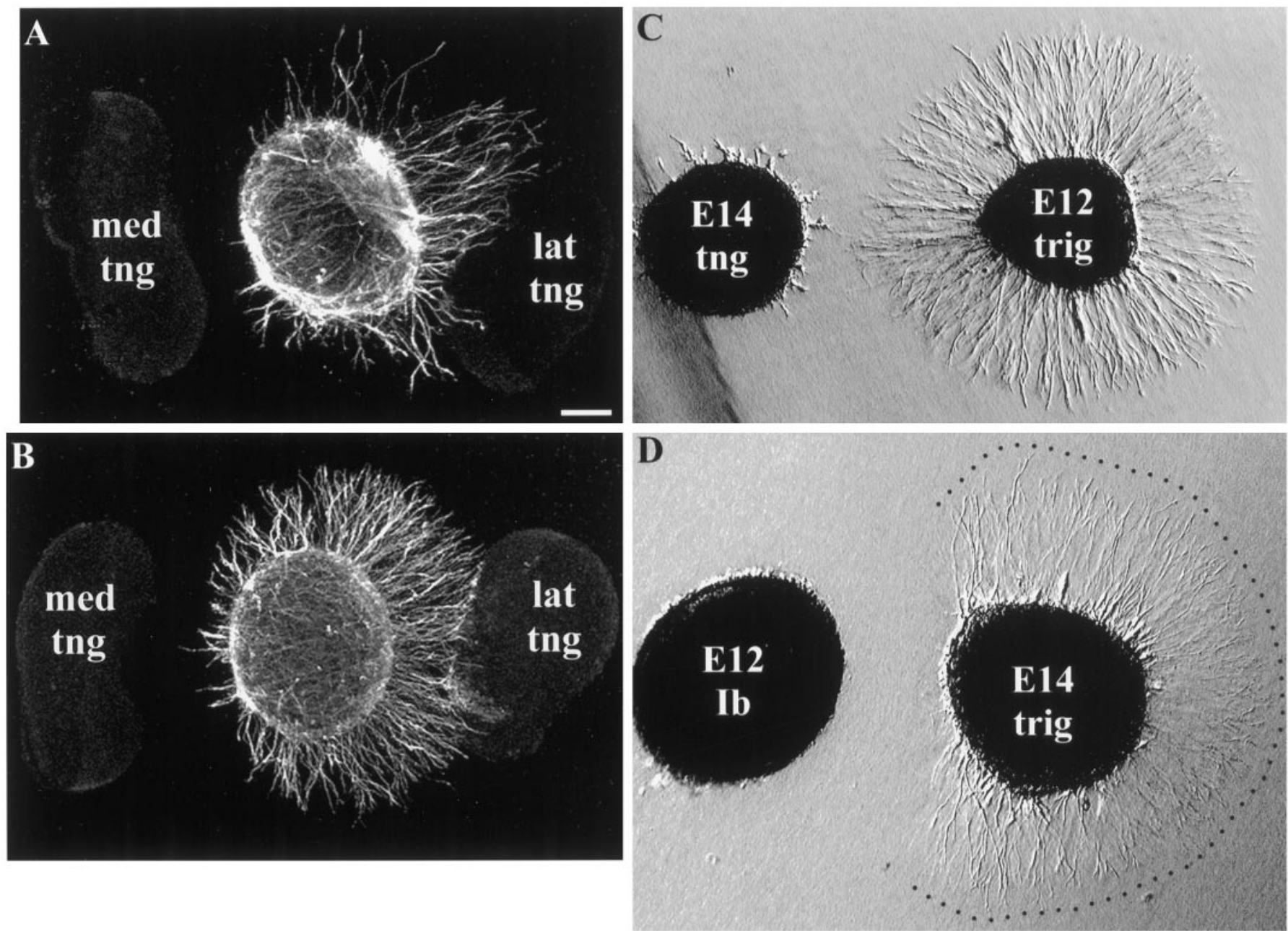

Figure 5. E14 dorsoanterior tongue has both attractive and repulsive territory. In the absence of added growth factors, trigeminal axon outgrowth preferentially grows toward lateral, rather than medial tongue explants $(A)$. In the presence of NGF $(2 \mathrm{ng} / \mathrm{ml})$, the medial tongue explant is avoided but not the lateral tongue $(B)$. Montages consist of a projection of a Z-series of confocal images taken with a $10 \times$ nonimmersion objective. Cultures were fixed after $18 \mathrm{hr}$ in vitro, labeled, and imaged as described in Materials and Methods. $C, D$, Net attraction to parasagittal sections of dorsal tongue is associated with a downregulation of repellant, not a downregulation of responsiveness to the repellant. $C$, E14 dorsoanterior tongue explant (excluding the septum) does not repel axon outgrowth stimulated by NT-4/5 (5 ng/ml) from an E12 posterior trigeminal ganglion explant ( $2 \mathrm{~d}$ in vitro $). D$, E12 arch Ib explant repels axon outgrowth stimulated by NGF $(2 \mathrm{ng} / \mathrm{ml})$ from an E14 posterior trigeminal ganglion explant $(1 \mathrm{~d}$ in vitro $)$. Scale bar, $100 \mu \mathrm{m}$.

anterior surfaces are repellant, axons growing anteriorly are sandwiched between two repellant surfaces (cf. Keynes et al., 1997) and may be induced to remain deep in the mesenchyme, as observed before E14.

The repellant also appears to be distributed in a mediolateral gradient, strongest in the medial mandible (Fig. 6, gray shaded areas). From E12 to E13, this gradient could restrict the earliest mandibular afferents away from medial structures, e.g., tongue. The inferior alveolar nerve turns from medial to anterior near mid-E13, avoiding the tongue, consistent with this possibility. Thereafter, on E14, the net repellant influence becomes restricted to a narrower medial territory. Presumably, the medial repellant influence and lateral attractant influence both contribute to the anterior radial turn of the first lingual nerve afferents. Note that chorda tympani fibers, which pioneer lingual sensory innervation, appear to attract lingual nerve afferents and could be their exclusive guidance influence. Nonetheless, geniculate ganglion axons are also repelled by medial arch Ib epithelium at E12 and E13 (Rochlin and Farbman, unpublished results), so regard- less of whether the lingual nerve afferents are restricted more by their preference for chorda tympani axons or by the repellant influence, the repellant influence likely contributes to their initial restriction away from the midline. Thus, our data suggest that repellant activity has a role in steering growth cones away from a medial course, and that the repellant influence becomes progressively restricted to more medial regions, thereby influencing the order and course of mandibular nerve branch development. This in turn may influence the timing of target contact.

Surprisingly, semaphorin III/D continues to be expressed throughout dorsoanterior tongue epithelium at least through E16 (Giger et al., 1996), a finding that underscores the importance of evaluating the repellant influence of tissues that express candidate repellants. The decreased repellant activity could be attributable to a reduction in repellant expression, an increase in a diffusible attractant, elevated furin proteolysis (Mark et al., 1997), or changes in the extracellular matrix that restrict its diffusion, but it is not attributable to decreased sensitivity to the repellant. If semaphorin III/D shifts from long-range repellant to 

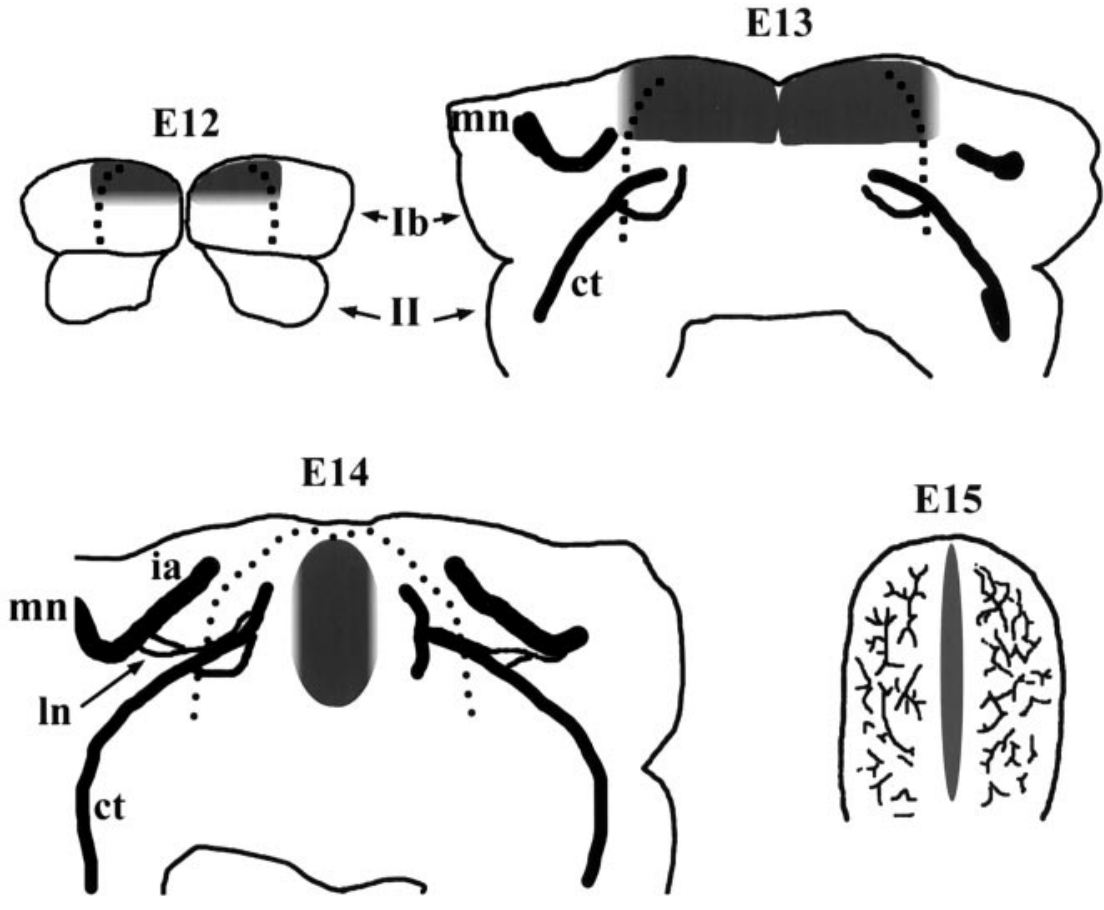

short-range repellant or stop signal, trophic and tropic factors secreted either from the epithelium or subepithelial mesenchyme could attract afferents to these peripheral targets. This possibility is particularly intriguing given that chorda tympani and lingual nerve afferents arrive in the tongue on E13 and E14, respectively, but do not penetrate the epithelium until E16 (Farbman and Mbiene, 1991).

The repellant sensitivity was independent of the neurotrophin used at the stages we studied, suggesting that all afferents are repellant-sensitive at these stages. At later stages, dorsal root ganglia (DRG) exhibit neurotrophin-dependent sensitivity (Mark et al., 1997). In particular, NT-3-stimulated DRG axon outgrowth becomes insensitive to semaphorin III/D, a condition that could permit NT-3-sensitive afferents to penetrate tissues that are repellant to axons responsive to other neurotrophins (Fitzgerald et al., 1993; Messersmith et al., 1995, Püschel et al., 1996; Shephard et al., 1997). Although NT-3 mRNA is present within tongue epithelium (Nosrat et al., 1996), NT-3-stimulated axon outgrowth from E16 trigeminal ganglia is repelled by E12 arch Ib explants (M. Rochlin, unpublished observations). Given the reported paucity of TrkC receptors in the E14 trigeminal ganglion (Elkabes et al., 1994), NT-3 may have been stimulating outgrowth via TrkA (Belliveau et al., 1997), which would not be expected to induce repellant-insensitive outgrowth. A subset of cutaneous trigeminal afferents are exclusively BDNF-supported (Buchman and Davies, 1993; Buj-Bello et al., 1994). It will be interesting to determine whether they innervate the tongue or exhibit repellant sensitivity at these stages. Given that the pattern of innervation of the tongue is initially specified by papillae differentiation (Farbman, 1965; Farbman and Mbiene, 1991; Whitehead and Kachele, 1994), which itself depends on neurotrophin signaling (Nosrat et al., 1997), and given that neurotrophins are topographically specialized in the tongue epithelium (Nosrat and Olson, 1995; Nosrat et al., 1996), it will be important to determine the distribution of repellant molecules at more mature stages and test for such an interaction.
Figure 6. Progressive narrowing of the chemorepellant region around the midline may influence the pattern and order of initial mandibular nerve branch development. Dorsal views of E12-E14 mandible and E15 tongue, traced from Figure 1, are shown; anterior is toward the top. Regions exhibiting chemorepellant activity are indicated by shading. Nerves were traced with thick black lines, except at E15, in which axon branches were traced with branched line segments. The borders of the developing tongue are indicated with dotted lines in the E12-E14 mandibles. The earliest mandibular nerve afferents enter arch Ib by E13. A repellant region is present before the arrival of these afferents (early E12, shaded region) and continues to be expressed by ventroanterior arch $\mathrm{Ib}$ through E13. This repellant influence may contribute to the anterior turn exhibited by the earliest afferents, those that contribute to the inferior alveolar branch ( $i a)$. Fortuitously, the turn can be appreciated in the E13 mandible by comparing the right and left mandibular nerves, which are at different developmental stages. By early E14, lingual nerve afferents have grown toward and joined the chorda tympani and entered the tongue. E14 medial tongue is still chemorepellant, but the lateral tongue is not chemorepellant and appears to be attractive. The lingual nerve afferents initially turn anteriorly and then grow radially toward the lateral tongue epithelia, which is consistent with a role for these diffusible cues in governing the distribution of lingual nerve afferents during development. Scale bar, $0.5 \mathrm{~mm}$.
We have shown that presumptive targets of sensory axons initially repel their afferents and do not have a net attractant influence until after the afferents have navigated to the vicinity of the target using intermediate cues. The repellant influence appears to contribute to the timing and coordination of growth cone advance toward presumptive targets, and is spatially and temporally regulated within a single tissue. Our data do not exclude a role for chemotropism in the initial guidance of trigeminal axons, but imply that short-range cues have a more important role than previously thought. There is evidence for short-range positive cues: trigeminal axons travel within a laminin-enriched core of mesenchyme in chick arch Ib (Riggott and Moody, 1987). Whether laminin(s) and chemoattractants are involved in the guidance of trigeminal axons at intermediate sites remains to be determined. In summary, a variety of factors, both long- and short-range, guide trigeminal axons to their targets during development, but the dominant long-range diffusible influence of some targets is repellant before becoming attractant.

\section{REFERENCES}

Adams RH, Betz H, Püschel AW (1996) A novel class of murine semaphorins with homology to thrombospondin is differentially expressed during early embryogenesis. Mech Dev 57:33-45.

Altman J, Bayer SA (1982) Development of the cranial nerve ganglia and related nuclei in the rat. Adv Anat Embryol Cell Biol 74:1-89.

Arumäe U, Pirvola U, Palgi J, Kiema TR, Palm K, Moshnyakov M, Ylikoski J, Saarma M (1993) Neurotrophins and their receptors in rat peripheral trigeminal system during maxillary nerve growth. J Cell Biol 122:1053-1065.

Belliveau DJ, Krivko I, Kohn J, Lachance C, Pozniak C, Rusakov D, Kaplan D, Miller FD (1997) NGF and neurotrophin-3 both activate TrkA on sympathetic neurons but differentially regulate survival and neuritogenesis. J Cell Biol 136:375-88.

Buchman VL, Davies AM (1993) Different neurotrophins are expressed and act in a developmental sequence to promote the survival of embryonic sensory neurons. Development 118:989-1001.

Buj-Bello A, Pinon LGP, Davies AM (1994) The survival of NGFdependent but not BDNF-dependent cranial sensory neurons is pro- 
moted by several different neurotrophins early in their development. Development 120:1573-1580.

Colamarino SA, Tessier-Lavigne M (1995) The axonal chemoattractant netrin-1 is also a chemorepellent for trochlear motor axons. Cell 81:621-9.

Davies AM (1997) Neurotrophin switching: where does it stand? Curr Opin Neurobiol 7:110-118.

Davies AM, Lumsden AG, Slavkin HC, Burnstock G (1981) Influence of nerve growth factor on the embryonic mouse trigeminal ganglion in culture. Dev Neurosci 4:150-156.

Ebendal T (1989) Use of collagen gels to bioassay nerve growth factor activity. In: Nerve growth factors (Rush RA, ed), pp 81-93. New York: Wiley.

Elkabes S, Dreyfus CF, Schaar DG, Black IB (1994) Embryonic sensory development: Local expression of Neurotrophin-3 and target expression of nerve growth factor. J Comp Neurol 341:204-213.

Ernfors P, Lee K-F, Jaenisch R (1994) Mice lacking brain-derived neurotrophic factor develop with sensory deficits. Nature 368:147-150.

Fan J, Raper JA (1995) Localized collapsing cues can steer growth cones without inducing their full collapse. Neuron 14:263-74.

Farbman AI (1965) Electron microscopic study of the developing taste bud in rat fungiform papilla. Dev Biol 11:110-135.

Farbman AI, Mbiene JP (1991) Early development and innervation of the taste bud-bearing papillae on the rat tongue. J Comp Neurol 306:172-186.

Fitzgerald M, Kwiat GC, Middleton J, Pini A (1993) Ventral spinal cord inhibition of neurite outgrowth from embryonic rat dorsal root ganglia. Development 117:1377-1384.

Flenniken AM, Gale NW, Yancopoulos GD, Wilkinson DG (1996) Distinct and overlapping expression patterns of ligands for Eph-related receptor tyrosine kinases during mouse embryogenesis. Dev Biol 179:382-401.

Gale NW, Holland SJ, Valenzuela DM, Flenniken A, Pan L, Ryan TE, Henkemeyer M, Strebhardt K, Hirai H, Wilkinson DG, Pawson T, Davis S, Yancoupolos GD (1996) Eph receptors and ligands comprise two major specificity subclasses and are reciprocally compartmentalized during embryogenesis. Neuron 17:9-19.

Giger RJ, Wolfer DP, de Wit MJ, Verhaagen J (1996) Anatomy of rat semaphorin III/collapsin-1 mRNA expression and relationship to developing nerve tracts during neuroembryogenesis. J Comp Neurol 375:378-392.

Gregg JM, Dixon AD (1973) Somatotopic organization of the trigeminal ganglion in the rat. Arch Oral Biol 18:487-498.

He H, Tessier-Lavigne M (1997) Neuropilin is a receptor for the axonal chemorepellant semaphorin III. Cell 90:739-751.

Hogg ID, Bryant JW (1969) The development of sensory innervation in the mouth and pharynx of the albino Norway rat (Mus norvegicus albinus). J Comp Neurol 136:33-56.

Keino-Masu K, Masu M, Hinck L, Leonardo ED, Chan SS, Culotti JG, Tessier-Lavigne M (1996) Deleted in Colorectal Cancer (DCC) encodes a netrin receptor. Cell 87:175-185.

Keynes R, Tannahill D, Morgenstern DA, Johnson AR, Cook GMW, Pini A (1997) Surround repulsion of spinal sensory axons in higher vertebrate embryos. Neuron 18:889-897.

Kitsukawa T, Shimizu M, Sanbo M, Hirata T, Taniguchi M, Bekku Y, Yagi T, Fujisawa H (1997) Neuropilin-semaphorin III/D-mediated chemorepulsive signals play a crucial role in peripheral nerve projection in mice. Neuron 19:995-1005.

Kobayashi H, Koppel AM, Luo Y, Raper JA (1997) A role for collapsin-1 in olfactory and cranial sensory axon guidance. J Neurosci 17:8339-8352.

Kolodkin AL, Levengood DV, Rowe EG, Tai Y-T, Giger RJ, Ginty DD (1997) Neuropilin is a semaphorin III receptor. Cell 90:753-762.

Leonardo ED, Hinck L, Masu M, Keino-Masu K, Ackerman SL, TessierLavigne M (1997) Vertebrate homologues of C. elegans UNC-5 are candidate netrin receptors. Nature 386:833-838.

Livesey FJ, Hunt SP (1997) Netrin and netrin receptor expression in the embryonic mammalian nervous system suggests roles in retinal, striatal, nigral, and cerebellar development. Mol Cell Neurosci 8:417-429.

Lumsden AGS (1982) The developing innervation of the lower jaw and its relation to the formation of tooth germs in mouse embryos. In: Teeth: form, function, and evolution (Kurtén B, ed), pp 32-43. New York: Columbia UP.

Lumsden AG, Davies AM (1983) Earliest sensory nerve fibres are guided to peripheral targets by attractants other than nerve growth factor. Nature 306:786-788.

Lumsden AG, Davies AM (1986) Chemotropic effect of specific target epithelium in the developing mammalian nervous system. Nature 323:538-539.

Luo Y, Raible D, Raper JA (1993) Collapsin: a protein in brain that induces the collapse and paralysis of neuronal growth cones. Cell 75:217-227.

Mark MD, Lohrum M, Püschel AW (1997) Patterning neuronal connections by chemorepulsion: the semaphorins. Cell Tissue Res 290:299-306.

Messersmith EK, Leonardo ED, Shatz CJ, Tessier-Lavigne M, Goodman CS, Kolodkin AL (1995) Semaphorin III can function as a selective chemorepellent to pattern sensory projections in the spinal cord. Neuron 14:949-959.

Mistretta CM, Haus LF (1996) Temporal and spatial patterns of tenascin and laminin immunoreactivity suggest roles for extracellular matrix in development of gustatory papillae and taste buds. J Comp Neurol 364:535-555.

Moody SA, Heaton MB (1983) Developmental relationships between trigeminal ganglia and trigeminal motoneurons in chick embryos. II. Ganglion axon ingrowth guides motoneuron migration. J Comp Neurol 213:344-349.

Nosrat CA, Olson L (1995) Brain-derived neurotrophic factor mRNA is expressed in the developing taste bud-bearing tongue papillae in rat. J Comp Neurol 360:698-704.

Nosrat CA, Ebendal T, Olson L (1996) Differential expression of brainderived neurotrophic factor and neurotrophin 3 mRNA in lingual papillae and taste buds indicates roles in gustatory and somatosensory innervation. J Comp Neurol 376:587-602.

Nosrat CA, Blomlof J, ElShamy WM, Ernfors P, Olson L (1997) Lingual deficits in BDNF and NT3 mutant mice leading to gustatory and somatosensory disturbances, respectively. Development 124:1333-1342.

Paves H, Arumäe U, Normet KJ, Saarma M (1994) Effects of Brainderived neurotrophic factor and neurotrophin-4 on embryonic rat sensory neurons. In: Life and death in the nervous system: role of neurotrophic factors and their receptors (Ibáñez CF, Fuxe K, Hökfelt T, Jörnvall H, Olson L, Ottoson D, eds) pp 191-202. Stockholm: WennerGren International Series.

Püschel AW, Adams RH, Betz H (1996) The sensory innervation of the mouse spinal cord may be patterned by differential expression of and differential responsiveness to semaphorins. Mol Cell Neurosci 7:419-431.

Riggott MJ, Moody SA (1987) Distribution of laminin and fibronectin along peripheral trigeminal axon pathways in the developing chick. J Comp Neurol 258:580-596.

Serafini T, Kennedy TE, Galko MJ, Mirzayan C, Jessell TM, TessierLavigne M (1994) The netrins define a family of axon outgrowthpromoting proteins homologous to C.elegans UNC-6. Cell 78:409-424.

Shepherd IT, Luo Y, Lefcort F, Reichardt LF, Raper JA (1997) A sensory axon repellent secreted from ventral spinal cord explants is neutralized by antibodies raised against collapsin-1. Development 124:1377-1385.

Stainier DYR, Gilbert W (1990) Pioneer neurons in the mouse trigeminal sensory system. Proc Natl Acad Sci USA 87:923-927.

Stainier DYR, Gilbert W (1991) Neuronal Differentiation and maturation in the mouse trigeminal sensory system, in vivo and in vitro. J Comp Neurol 311:300-312.

Taniguchi M, Yuasa S, Fujisawa H, Naruse I, Saga S, Mishina M, Yagi T (1997) Disruption of Semaphorin III/D gene causes severe abnormality in peripheral nerve projection. Neuron 19:519-530.

Tessier-Lavigne M, Goodman CS (1996) The molecular biology of axon guidance. Science 274:1123-1133.

Varela-Echavarria A, Tucker A, Püschel AW, Guthrie S (1997) Motor axon subpopulations respond differentially to the chemorepellants netrin-1 and semaphorin D. Neuron 18:193-207.

Whitehead MC, Kachele DL (1994) Development of fungiform papillae, taste buds, and their innervation in the hamster. J Comp Neurol 340:515-530.

Wright DE, White FA, Gerfen RW, Silos-Santiago I, Snider WD (1995) The guidance molecule semaphorin III is expressed in regions of spinal cord and periphery avoided by growing sensory axons. J Comp Neurol 361:321-333. 